\title{
High prevalence of multi drug resistant tuberculosis in people living with HIV in Western India
}

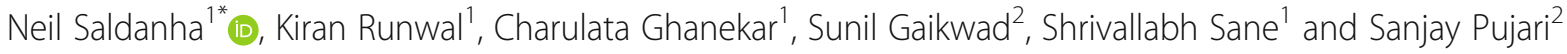

\begin{abstract}
Background: Most studies assessing drug resistant tuberculosis (DRTB) in human immunodeficiency virus (HIV) co-infected patients in India have used conventional culture- based systems to diagnose DRTB that have a longer turnaround time leading to risk of amplification of resistance to an empirical regimen. We determined the prevalence of DRTB amongst people living with HIV (PLHIV) using the line probe assay and determined risk factors associated with the presence of multi drug resistant tuberculosis (MDRTB).

Methods: A Cross-sectional study was undertaken at Poona Hospital and Research Center (PHRC) and the Institute of Infectious Diseases, two tertiary level private care centers in Pune, India. Consenting PLHIV with confirmed Pulmonary TB (PTB) and/or extra-pulmonary TB (EPTB) diagnosed based on detection of Mycobacterium TB by line probe assay (Geno Type MTBDRplus version 2) on clinical specimens were included. Those with documented past history of DRTB were excluded. Resistance against anti-TB drugs was determined by the same assay. The prevalence of any form of drug resistant TB (DRTB), MDRTB, Rifampicin resistant TB (RRTB) and Isoniazid (INH) mono-resistant TB were determined as the proportion of these amongst all included PLHIV-TB. A multivariate analysis was conducted to determine risk factors that were statistically associated with MDRTB, DRTB, RRTB and INH mono-resistant TB.

Results: Two hundred PLHIV were recruited. The prevalence $(95 \% \mathrm{CI})$ of MDRTB, INH mono- resistance and RR resistance was $12.5 \%(7.9-17.1 \%), 9 \%(6.9-11.2 \%)$ and $2.5 \%(1.4-3.6 \%)$, respectively. The prevalence (95\% Cl) of MDRTB among new and relapsed patients was $8.8 \%$ (6.5-11.1\%) and 23.1\% (17.2-28.9\%), respectively. Tuberculosis relapse was the only factor significantly associated with MDRTB, DRTB and INH mono-resistant TB.

Conclusion: We document a high prevalence of drug resistance to anti-TB drugs including MDRTB among PLHIV in our setting using Geno Type MTBDRplus directly on clinical specimens. This validates the WHO recommendation of performing routine rapid molecular resistance testing prior to initiating anti-TB treatment among all PLHIV with presumptive TB. Using rapid molecular testing especially Geno Type MTBDRplus (that detects resistance to INH and Rifampicin simultaneously) reduces the turn-around time helping in optimizing treatment.
\end{abstract}

Keywords: MDRTB, HIV, India, Prevalence

\footnotetext{
* Correspondence: drneilsaldanha@gmail.com

${ }^{1}$ Consultant Biostatistician, Poona Hospital and Research Center, Pune, India

Full list of author information is available at the end of the article
}

(c) The Author(s). 2019 Open Access This article is distributed under the terms of the Creative Commons Attribution 4.0 International License (http://creativecommons.org/licenses/by/4.0/), which permits unrestricted use, distribution, and reproduction in any medium, provided you give appropriate credit to the original author(s) and the source, provide a link to the Creative Commons license, and indicate if changes were made. The Creative Commons Public Domain Dedication waiver (http://creativecommons.org/publicdomain/zero/1.0/) applies to the data made available in this article, unless otherwise stated. 


\section{Background}

Tuberculosis (TB) is one of the most common opportunistic infections in people living with human immunodeficiency virus (PLHIV) and a major contributor to morbidity and mortality [1, 2]. Drug-resistant TB (DRTB), including multi-drug resistant TB (MDRTB), defined as resistance to isoniazid (INH) and rifampicin (Rif), is rapidly emerging and threatens to derail the success of TB control programs in high endemic TB settings [3]. Although HIV infection by itself does not increase the risk of acquiring primary DRTB, using intermittent treatment for TB has been associated with the development of acquired rifampicin resistance [4].

According to the Global Tuberculosis Report 2018, India has the highest burden of TB (27\%) and MDRTB (24\%) in the world [5]. In 2017, estimated proportion of MDRTB amongst new and previously treated cases were 2.0 and $12 \%$ respectively in India.

An estimated 9\% of incident TB cases in 2017 were amongst PLHIV globally. Few studies in India have explored the prevalence of DRTB among PLHIV [6, 7]. In a study amongst PLHIV attending a public sector Antiretroviral center in Mumbai, the prevalence of MDRTB (drug susceptibility testing using phenotypic liquid culture) was $38 \%$ and only previous history of TB was significantly associated with the diagnosis of DRTB. An MDRTB rate of $5.6 \%$ was reported amongst PLHIV from South India, however Geno Type MTBDRplus for diagnosis was done retrospectively on culture isolates [7] and risk factors were not explored.

The World Health Organization (WHO) recommends baseline resistance testing prior to initiating an anti-Tuberculous treatment (ATT) for all PLHIV with active TB. The line probe assay effectively detects INH and Rif resistance rapidly and has an advantage over the Xpert TB/Rif that detects only Rif resistance [8]. Information on prevalence and risk factors for MDRTB (diagnosed by rapid molecular tests on clinical specimens) amongst PLHIV is urgently needed in India. We determined the prevalence of DR-TB among PLHIV using the line probe assay and determined risk factors associated with the presence of MDRTB.

\section{Methods}

\section{Design and study site}

The study employed a cross-sectional design and was conducted between Jan 2014 and Dec 2016. Two tertiary level care centers, Poona Hospital and Research Center (PHRC) and the Institute of Infectious Diseases (IID) in Pune, Western India participated. Both these centers are urban and situated in the same city. While IID is an out-patient clinic providing comprehensive care to PLHIV and other infectious diseases, PHRC provides in-patient and laboratory support to IID. The study protocol was approved by the Independent Ethics Committee of PHRC.

\section{Patients}

All consenting PLHIV with confirmed pulmonary (PTB) and/or extra-pulmonary TB (EPTB) were consecutively recruited in the study. Confirmation was based on detection of mycobacterium TB (MTB) in clinical specimens by Line Probe Assay (Genotype MTBDRplus). For PTB, sputum or Bronchoscopic Alveolar lavage (BAL) were processed. Extrapulmonary TB was presumably diagnosed based on histo-pathologic evidence of classical caseating granulomas in clinical specimens like lymph nodes and confirmed with detection of MTB on Geno Type MTBDRplus. Those with a past history of documented DRTB were excluded.

\section{Procedures}

A written consent was provided by all PLHIV participating in the study. Data collection and documentation was done using a standardized case report form by the principal investigator.

Demographic information including HIV related (duration since diagnosis, current, and past CD4 counts, and viral loads), ART (antiretroviral therapy) related (duration on ART, type of regimen, whether failing) and TB related (pulmonary/extrapulmonary, new/treatment cases) were collected from past medical records and self-report. HIV infection was diagnosed based on a positive result on 4th generation ELISA and rapid immunochromatography. Resistance to anti-TB drugs was determined by the line probe assay Geno Type MTBDRplus version 2, (HAIN Lifesciences $\mathrm{GmbH}$, Hardwiesenstraße, Nehren, Germany). In this assay, the resistance to INH and Rif is determined by identifying mutations in the INH A/Kat $G$ and $r p o B$ genes, respectively. The plasma viral load was determined by COBAS TaqMan (Roche Molecular Systems, Branchburg, NJ), while CD4 counts were determined by FACSCount (BD Biosciences, Sane Jose, CA, USA).

We defined MDRTB as resistance to INH and Rif, RRTB as resistance to Rif only, and DRTB as either mono-resistance (RRTB or INH mon-resistance) or MDRTB. Primary TB was defined as diagnosed with TB for the first time and never treated with anti-TB drugs while TB relapse was defined as patients who completed a course of anti-TB treatment in the past.

\section{Statistical analysis}

The prevalence of MDRTB was determined by calculating the proportion of patients with MDRTB among those with TB. The significance of differences among the demographic variables between the two groups (MDRTB versus non-MDRTB) was determined by Mann-Whitney 
test and chi-square test for continuous and categorical variables, respectively.

Multivariate analyses (logistic regression) were used to determine statistically significant risk factors for the presence of MDRTB, DRTB, RRTB and INH mono-resistant TB. The outcome variable was treated as dichotomous variable (MDRTB vs non-MDRTB, DRTB vs non-DRTB). The exploratory variables included age, gender, duration since HIV diagnosis, current CD4 counts, and PVL, type of TB (PTB and EPTB), primary and relapsed TB. A $p$-value of less than 0.05 was considered statistically significant.

A sample size of 200 was calculated assuming a prevalence of $15 \%$ for MDRTB amongst relapsed cases with $90 \%$ power and a p-value of 0.05 . All analyses were done using SPSS version 20 software.

\section{Results}

Two hundred patients with confirmed TB were recruited. The prevalence (95\% CI) of MDRTB was $12.5 \%$ (7.9-17.1\%). Prevalence of INH mono-resistance, RR and DRTB was 9.0\% (6.9-11.2\%) and 2.5\% (1.4-3.6\%), and $24.0 \%(20.9-27.0 \%)$ respectively. Sixty-two percent of the drug resistance to INH was mediated through KatG mutations.

The demographics for patients with TB, MDRTB, and non-MDRTB are summarized in Table 1.

The median age (range) of all TB patients was 41.5 (11-48) years and $67 \%$ were males. The median duration (range) since HIV diagnosis was 5 [3-10] years. The median (range) current CD4 count was $114(9-644) / \mathrm{mm}^{3}$, while approximately $74.5 \%$ patients had a CD4 count less than $200 / \mathrm{mm}^{3}$. Sixty percent of patients were on ART (antiretroviral therapy) and $68.0 \%$ of patients were diagnosed to have EPTB. Lymphadenopathy (63.0\%) constituted the major form of EPTB. Seventy-four percent of patients had primary TB. Among relpased cases 48.1\% had previous PTB. Most patients (92.5\%) with TB were smear positive.

The prevalence of MDRTB in PLHIV with CD4< $200 / \mathrm{mm}^{3}$ was higher $(14.0 \%)$ than those with $\mathrm{CD} 4>$ $200 / \mathrm{mm}^{3}$ (5.8\%), although this difference was not statistically significant. However, PLHIV with MDRTB had a lower median CD4 count $\left(90 / \mathrm{mm}^{3}\right)$ as compared to non-MDRTB $\left(120 / \mathrm{mm}^{3}, p=0.04\right)$. In multivariate analyses, only previously treated cases had higher odds for the presence of MDRTB (Table 2). The prevalence (95\% CI) of MDRTB among new and relapsed patients was $8.8 \%(6.5-11.1 \%)$ and $22.6 \%(11.3-33.9 \%)$, respectively. The prevalence $(95 \% \mathrm{CI})$ of INH mono-resistance in the new and relapsed patients was $7.4 \%(3.8-14.9 \%)$ and $13.5 \%(8.7-18.1 \%)$, respectively, while that of rifampicin mono-resistance was $2.7 \%(0.5-3.6 \%)$ and $1.9 \%(0.1-$ $3.82 \%)$, respectively, and DRTB was $18.9 \%$ (12.6-25.2\%) and $38.5 \%$ (25.2-51.7\%), respectively. In multivariate analysis, only retreatment was significantly associated with higher odds of DRTB, MDRTB and INH mono-resistance, 2.4 (1.2-4.9), 2.5 (1.0-6.3) and $2.6(1.2-5.5)$, respectively (Table 2).

\section{Discussion}

We have documented a high prevalence of overall drug resistance against anti-TB drugs including MDRTB in PLHIV. This is higher than the WHO's global estimate of 3.5 and $20.5 \%$ MDRTB among new and retreatment cases respectively, among all $\mathrm{TB}$ cases irrespective of HIV infection status [9]. However, the prevalence of MDRTB in this study is similar to those documented by a few other studies in India. For instance, a study on PLHIV attending public ART program in Mumbai

Table 1 Demographics of PLHIV with all TB, MDRTB and non-MDRTB

\begin{tabular}{|c|c|c|c|c|}
\hline & All TB $(n=200)$ & MDRTB $(n=25)$ & Non-MDRTB $(n=175)$ & $P$ value (MDRTB vs Non-MDRTB) \\
\hline \multicolumn{5}{|l|}{ Age, years } \\
\hline Median (range) & $41.5(11-48)$ & $43(11-65)$ & $40(16-79)$ & 0.70 \\
\hline Male sex, n (\%) & $134(67.0)$ & $18(72.0)$ & $116(66.2)$ & 0.56 \\
\hline \multicolumn{5}{|c|}{ Duration since HIV diagnosis, years } \\
\hline Median (range) & $5(1-23)$ & $4(1-19)$ & $5(1-23)$ & 0.21 \\
\hline \multicolumn{5}{|l|}{ CD4 counts } \\
\hline Median (range) & $114(9-644)$ & $90(11-591)$ & $120(9-644)$ & 0.04 \\
\hline \multicolumn{5}{|l|}{ ART status } \\
\hline Naïve, n (\%) & 79 (39.5) & $11(44.0)$ & 68 (38.9) & 0.62 \\
\hline Experienced, n (\%) & $121(60.5)$ & $14(56.0)$ & $107(61.1)$ & \\
\hline \multicolumn{5}{|l|}{ TB } \\
\hline Primary, n (\%) & $147(73.5)$ & $13(52.0)$ & $135(77.1)$ & 0.007 \\
\hline Relapsed, n (\%) & $53(26.5)$ & $12(48.0)$ & $40(22.8)$ & \\
\hline
\end{tabular}


Table 2 Multivariate analysis to explore risk factors for presence of DRTB, MDRTB, RRTB and INH mono-resistance

\begin{tabular}{|c|c|c|c|c|}
\hline & $\begin{array}{l}\text { DRTB } \\
\text { OR }(95 \% \mathrm{Cl})\end{array}$ & $\begin{array}{l}\text { MDRTB } \\
\text { OR }(95 \% \mathrm{Cl})\end{array}$ & $\begin{array}{l}\text { RRTB } \\
\text { OR }(95 \% \mathrm{Cl})\end{array}$ & $\begin{array}{l}\text { INH mono-resistance } \\
\text { OR }(95 \% \mathrm{Cl})\end{array}$ \\
\hline \multicolumn{5}{|l|}{$\overline{A g e}<30$} \\
\hline Age $31-60$ & $1.0(0.2-4.3)$ & $0.4(0.1-4.5)$ & $0.3(0.1-2.9)$ & $1.4(0.3-6.0)$ \\
\hline Age $>60$ & $1.2(0.3-4.4)$ & $0.4(0.1-3.9)$ & $0.4(0.1-3.3)$ & $1.4(0.4-5.1)$ \\
\hline Gender & $1.0(0.5-2.1)$ & $1.4(0.5-3.6)$ & $1.5(0.6-3.7)$ & $0.9(0.4-1.9)$ \\
\hline \multicolumn{5}{|l|}{ HIV-Duration $<5$} \\
\hline $6-10$ years & $0.8(0.3-2.3)$ & $0.6(0.2-2.4)$ & $0.7(0.3-2.3)$ & $0.8(0.2-2.4)$ \\
\hline$>10$ years & $1.6(0.5-4.7)$ & $1.1(0.3-5.1)$ & $1.3(0.5-4.7)$ & $1.5(0.3-4.9)$ \\
\hline \multicolumn{5}{|l|}{ CD4 counts $<50$} \\
\hline $51-100$ & $0.6(0.2-1.8)$ & $0.2(0.1-1.2)$ & $0.3(0.1-1.2)$ & $0.6(0.2-1.8)$ \\
\hline$>100$ & $0.7(0.3-1.6)$ & $0.5(0.1-1.2)$ & $0.5(0.1-1.5)$ & $0.7(0.3-1.7)$ \\
\hline ART status & $1.0(0.5-2.4)$ & $1.1(0.4-3.2)$ & $1.2(0.5-3.1)$ & $1.0(0.4-2.4)$ \\
\hline Type of TB: EPTB & $0.6(0.3-1.3)$ & $0.5(0.1-1.2)$ & $0.6(0.2-1.4)$ & $0.6(0.3-1.2)$ \\
\hline Primary and relapse & $2.4(1.2-4.9)^{*}$ & $2.5(1.0-6.3)^{* *}$ & $2.18(1.0-5.1)$ & $2.6(1.2-5.5)^{* * *}$ \\
\hline
\end{tabular}

${ }^{*} p=0.018,{ }^{* *} p=0.048,{ }^{* * *} p=0.012$

The outcome variable was treated as dichotomous variable (MDRTB vs non-MDRTB, DRTB vs non-DRTB, RRTB vs non-RRTB and INH mono-resistant TB vs non-INH mono-resistant TB)

Odds Ratio (OR)

documented a MDRTB prevalence of $11.4 \%$ amongst new TB cases and $36.4 \%$ amongst re-treated TB cases [6]. However, the study was predominantly conducted among PLHIV belonging to lower socio-economic strata and resistance was determined using phenotypic liquid culture. We did not formally assess the socio-economic status of our patients. However, our centers are private care setups and patients accessing care pay out of pocket (middle to higher socio-economic status) unlike the public setup of the Mumbai study that provides diagnosis and treatment free. In another study from a TB control program clinic in Delhi, an MDRTB prevalence of $13.2 \%$ was documented using phenotypic methods [10].

A prevalence of MDRTB of 7\%, using the line probe assay on culture isolates, was documented in a study from South India [11]. The sample size in this study was small, only 76 mycobacterial strains obtained from 67 patients. Prevalence of Rif resistant TB was $11.2 \%$, by Xpert MTB/RIF, amongst PLHIV with TB $(n=867)$ participating in a large demonstration study across public clinics in India [12]. To the best of our knowledge, this is the largest study determining the prevalence of DRTB including MDRTB using line probe assay on clinical specimens in PLHIV in India. This provides a more robust estimate of the burden of DRTB/MDRTB in PLHIV, although it is valid only for those availing medical care in the private sector.

It is crucial to identify DRTB/MDRTB rapidly to improve clinical outcomes and prevent further transmission to the contacts. Traditional culture and susceptibility methods have a long turnaround time (up to 4-6 weeks) that delays the initiation of appropriate anti-TB regimen
[13]. The turnaround time for rapid molecular tests varies from two hours to two days facilitating early treatment and preventing further transmission thus having a potential to have a major public health impact [12]. The performance of these tests has been comparable to the traditional culture and phenotypic susceptibility methods [14-16]. We did not simultaneously perform culture and susceptibility on our specimens and hence cannot comment on the accuracy of line probe assay. Historically, other studies from India have documented a higher prevalence of DRTB with phenotypic methods. Smear gradation appears to influence the probability of obtaining an interpretable result and the sensitivity of the line probe assay, indicating a significant association between the bacillary load and the test performance [14]. Heteroresistance may also explain this discordance, although this is unlikely to be true in our study.

We found no HIV related factors associated with higher odds for the presence of MDRTB in our study on multivariate analysis. However, on univariate analysis lower median CD4 count was associated with presence of MDRTB. There is conflicting evidence on HIV infection as a risk factor for the presence or development of MDRTB. In a Bayesian modeling study, HIV co-infection did not significantly affect the transmissibility and the mutation rate of MTB in patients and was not associated with the increased emergence of resistance [17].

Most of the studies have documented a higher prevalence of MDRTB in the retreated cases in PLHIV as well as those without HIV infection [6, 18]. Even in our study, the only risk factor associated with presence of MDRTB was TB relapse. Various factors may contribute 
to the higher risk of MDRTB in the retreated patients. Exposure to sub-optimal anti-TB regimens during previous treatment due to poor compliance, improper prescriptions, and drug storage and quality, may significantly contribute to the emergence of DRTB [19]. Apart from this, the patients may be treated with irrational fixed-dose combinations not adjusted to appropriate body weight. Intermittent $\mathrm{TB}$ treatment as used in the program has been associated with a higher risk of acquired rifampicin resistance, especially among PLHIV. Among PLHIV receiving thrice weekly anti-TB regimen, a higher incidence of acquired rifampicin resistance has been documented in Chennai, India [4]. While ART was associated with the lower presence of MDRTB in this study, it did not eliminate this risk completely. Finally, many previously treated patients used standard first-line WHO regimen without baseline resistance testing. In the context of undiagnosed INH resistance, further amplification of resistance to other drugs especially rifampicin may occur [20-22].

There are a few limitations of our study. We did not recruit concurrent HIV negative controls for demonstrating differences in the prevalence between the two groups as we did not have access to HIV negative patients with TB. We did not simultaneously perform phenotypic testing for detecting resistance and neither performed Genotype MTB DR plus/sl to detect resistance to ethambutol, fluoroquinolones, and aminoglycosides. Finally, our patients were belonging to the middle to high socio-economic strata and accessed care in the private health sector thus limiting external validity of our study.

\section{Conclusion}

We have documented a high prevalence of DRTB/ MDRTB in PLHIV with the line probe assay. This validates the WHO recommendation of performing routine rapid molecular resistance testing prior to initiating an anti-TB treatment in all PLHIV. These tests can also be performed on smear negative and directly on other extra-pulmonary specimens. Further studies are needed to confirm these findings, especially among PLHIV with $\mathrm{TB}$ accessing care in the public programs in India.

\section{Abbreviations \\ ATT: Anti-Tuberculous treatment; BAL: Bronchoscopic Alveolar lavage; DRTB: Drug resistant tuberculosis; EPTB: Extra-pulmonary TB; HIV: Human Immunodeficiency Virus; IID: Institute of Infectious Diseases; INH: Isoniazid; MDRTB: Multi drug resistant tuberculosis; MTB: Mycobacterium tuberculosis; PHRC: Poona Hospital and Research Center; PLHIV: People living with HIV; PTB: Pulmonary TB; Rif: Rifampicin; RRTB: Rifampicin resistant TB; TB: Tuberculosis; WHO: World Health Organization}

\section{Acknowledgments}

We thank all patients for their participation and the staff of Poona Hospital and Research Center and the Institute of Infectious Diseases for their timely help in the study.

\section{Funding}

No funding was received for the study.

Availability of data and materials

Raw data are available with the corresponding author.

\section{Authors' contributions}

NS- Designing the protocol, recruitment of patients, Collection of data and writing of the manuscript. KR- Concept and reviewing the manuscript. CGPerformed all microbiological laboratory investigations. SG- Recruitment of patients, writing, and reviewing the manuscript. SS- Statistical analysis of data. SP- Designing the protocol, concept, recruitment of patients, writing and revising manuscript as well as data planning and analysis. All authors have read and approved the manuscript.

\section{Ethics approval and consent to participate}

The study protocol was approved by the Independent Ethics committee of Poona Hospital and Research Center. Written informed consent was provided by all participants prior to study entry. The consent form was approved by the Independent Ethics Committee of Poona Hospital and Research Center. In case of children ( $<18$ years) the parents/guardian were informed in detail about the study and they provided written informed consent.

\section{Consent for publication}

Not applicable

\section{Competing interests}

The authors declare that they have no competing interests.

\section{Publisher's Note}

Springer Nature remains neutral with regard to jurisdictional claims in published maps and institutional affiliations.

\section{Author details}

${ }^{1}$ Consultant Biostatistician, Poona Hospital and Research Center, Pune, India. ${ }^{2}$ Institute of Infectious Diseases, Pune, India.

Received: 18 July 2018 Accepted: 29 April 2019

Published online: 08 May 2019

\section{References}

1. Tornheim J, Dooley K. Tuberculosis Associated with HIV Infection. Microbiol Spectrum. 2017:5(1):TNMI7-0028-2016. https://doi.org/10.1128/microbiolspec TNMI7-0028-2016

2. Adamu AL, Gadanya MA, Abubakar IS, Jibo AM, Bello MM, Gajida AU, et al High mortality among tuberculosis patients on treatment in Nigeria: a retrospective cohort study. BMC Infect Dis. 2017;17(1):170.

3. Hoffner S. Multidrug-resistant tuberculosis: the problem and some priorities in controlling it. Int J Mycobacteriol. 2016:5(Suppl 1):S59.

4. Narendran G, Menon PA, Venkatesan P, Vijay K, Padmapriyadarsini C, Ramesh Kumar $\mathrm{S}$, et al. Acquired rifampicin resistance in thrice-weekly antituberculosis therapy: impact of HIV and antiretroviral therapy. Clin Infect Dis. 2014;59(12):1798-804.

5. World Health Organisation. Global Tuberculosis report, https://www.who.int/ tb/publications/global_report/en/. Accessed Jan 2019.

6. Isaakidis P, Das M, Kumar AM, Peskett C, Khetarpal M, Bamne A, et al. Alarming levels of drug-resistant tuberculosis in HIV-infected patients in metropolitan Mumbai, India. PLoS One. 2014:9(10):e110461.

7. Iseki K. Gender differences in chronic kidney disease. Kidney Int. 2008;74(4): 415-7.

8. Kipiani M, Mirtskhulava V, Tukvadze N, Magee M, Blumberg HM, Kempker RR. Significant clinical impact of a rapid molecular diagnostic test (genotype MTBDRplus assay) to detect multidrug-resistant tuberculosis. Clin Infect Dis. 2014;59(11):1559-66.

9. Velayati AA, Farnia P, Farahbod AM. Overview of drug-resistant tuberculosis worldwide. Int J Mycobacteriol. 2016;5(Suppl 1):S161.

10. Magee MJ, Blumberg HM, Broz D, Furner SE, Samson L, Singh S, et al. Prevalence of drug resistant tuberculosis among patients at high-risk for Hiv attending outpatient clinics in Delhi. India Southeast Asian J Trop Med Public Health. 2012;43(2):354-63. 
11. Shivaswamy U, Neelambike SM. Drug resistance pattern of mycobacterial isolates in HIV and non-HIV population in South India. Lung India. 2016;33(1):27-31.

12. Raizada N, Sachdeva KS, Sreenivas A, Kulsange S, Gupta RS, Thakur R, et al. Catching the missing million: experiences in enhancing TB \& DR-TB detection by providing upfront Xpert MTB/RIF testing for people living with HIV in India. PLoS One. 2015;10(2):e0116721.

13. Nair D, Navneethapandian PD, Tripathy JP, Harries AD, Klinton JS, Watson B, et al. Impact of rapid molecular diagnostic tests on time to treatment initiation and outcomes in patients with multidrug-resistant tuberculosis, Tamil Nadu, India. Trans R Soc Trop Med Hyg. 2016;110(9):534-41.

14. Seifert M, Ajbani K, Georghiou SB, Catanzaro D, Rodrigues C, Crudu V, et al. A performance evaluation of MTBDRplus version 2 for the diagnosis of multidrug-resistant tuberculosis. Int J Tuberc Lung Dis. 2016;20(5):631-7.

15. Ali RM, Alsudani AA. Discordance between GeneXpert assay and conventional drug-susceptibility testing in detecting rifampicin-resistant tuberculosis: a perspective of the line probe assay. Int J Mycobacteriol. 2016;5(Suppl 1):S193-S4

16. Kotwal A, Biswas D, Raghuvanshi S, Sindhwani G, Kakati B, Sharma S. Diagnostic performance of automated liquid culture and molecular line probe assay in smear-negative pulmonary tuberculosis. Trop Doct. 2017; 47(2):118-23.

17. Eldholm V, Rieux A, Monteserin J, Lopez JM, Palmero D, Lopez B, et al. Impact of HIV co-infection on the evolution and transmission of multidrugresistant tuberculosis. Elife. 2016;5.

18. Munje R, Deshmukh $R$, Tumane K. Multidrug-resistant TB among previously treated TB cases: a retrospective study in Nagpur, India. Indian J Tuberc. 2015;62(4):207-10.

19. Rakesh PS, Balakrishnan S, Jayasankar S, Asokan RV. TB management by private practitioners - is it bad everywhere? Indian J Tuberc. 2016;63(4):251-4.

20. Gegia M, Winters N, Benedetti A, van Soolingen D, Menzies D. Treatment of isoniazid-resistant tuberculosis with first-line drugs: a systematic review and meta-analysis. Lancet Infect Dis. 2017;17(2):223-34.

21. Stagg HR, Lipman MC, McHugh TD, Jenkins HE. Isoniazid-resistant tuberculosis: a cause for concern? Int J Tuberc Lung Dis. 2017:21(2):129-39.

22. Arora J, Singhal R, Verma AK, Kumar G, Bhalla M, Sarin R, et al. Isoniazid resistance among rifampicin-susceptible Mycobacterium tuberculosis isolates from tuberculosis patients. Int J Mycobacteriol. 2016;5(Suppl 1):S127-S8.

Ready to submit your research? Choose BMC and benefit from:

- fast, convenient online submission

- thorough peer review by experienced researchers in your field

- rapid publication on acceptance

- support for research data, including large and complex data types

- gold Open Access which fosters wider collaboration and increased citations

- maximum visibility for your research: over $100 \mathrm{M}$ website views per year

At $\mathrm{BMC}$, research is always in progress.

Learn more biomedcentral.com/submissions 